\title{
DIRECTED ALGEBRAIC TOPOLOGY, CATEGORIES AND HIGHER CATEGORIES
}

\author{
MARCO GRANDIS
}

\begin{abstract}
Directed Algebraic Topology is a recent field, deeply linked with Category Theory. A 'directed space' has directed homotopies (generally non reversible), directed homology groups (enriched with a preorder) and fundamental $n$-categories (replacing the fundamental $n$ groupoids of the classical case). On the other hand, directed homotopy can give geometric models for lax higher categories. Applications have been mostly developed in the theory of concurrency. Unexpected links with noncommutative geometry and the modelling of biological systems have emerged.
\end{abstract}

\section{INTRODUCTION}

Directed Algebraic Topology (DAT) studies 'directed spaces' in some sense, where paths and homotopies cannot generally be reversed; for instance: ordered topological spaces, 'spaces with distinguished paths', 'inequilogical spaces', or also classical combinatorial structures like simplicial and cubical sets. Present applications of DAT deal mostly with the analysis of concurrent processes: see, for instance, [Go, Ga, FRGH] and the papers by E. Goubault - E. Haucourt and M. Raussen in this volume [GH, Ra]; another field is emerging, in the domain of rewriting systems, see Y. Lafont's article [La] also in the present issue. But the natural range of DAT should cover non reversible phenomena, in any domain.

Here, after a review of a series of papers devoted to this subject ([G4] to [G11]), we shall give some hints at future developments and new interactions with other domains. A wider literature can be found in the papers mentioned above.

Directed spaces can be studied with directed versions of the classical tools of Algebraic Topology. Thus, the directed homology groups $\uparrow H_{n}(X)$ (Sections 2-3, [G5, G7]) are preordered abelian groups. Similarly, the fundamental category $\uparrow \Pi_{1}(X)$ (Sections 4-6, [G4, G8]) replaces the classical fundamental groupoid; it also allows one to study situations where all directed loops are constant (so that the fundamental monoids are trivial, and $\uparrow H_{1}(X)$ is reduced to its algebraic part, with the discrete order). The study of higher

1991 Mathematics Subject Classification. 55Pxx, 18D05, 68Q85.

Key words and phrases. directed algebraic topology, homotopy theory, 2-categories, lax categories, fundamental $n$-category, concurrent processes.

Work partially supported by MIUR Research Projects. 
fundamental categories, begun in a strict 2-dimensional version [G9], is now progressing in lax versions [G10, G11], which seem to be more natural and adapted to higher extensions (Section 7).

DAT has thus a deep interaction with ordinary and higher dimensional category theory, clearer than classical Algebraic Topology. Strong connections with Noncommutative Geometry have already been developed (Section 3, [G5, G6, G7, G12]); these deal with parallel realisations - in Noncommutative Geometry and DAT - of orbit spaces and spaces of leaves which are trivial in ordinary topology. Other similarities have recently appeared, between the notion of root of a category developed by A.C. Ehresmann [Eh] for modelling biological systems, and our study of the fundamental category (Section 8).

Section 9 contains a review of effective topological settings for DAT, after the combinatorial ones and the basic topological setting of (pre)ordered spaces previously considered. Then, we examine some initial steps for an axiomatic setting (Sections 10, 11), based on abstracting the (co)cylinder functor and its natural transformations, as in Kan's approach to homotopy [Ka]. (Quillen's model structures are used in [Ga], even if they do not allow to formalise directed homotopies.) We end with some hints to a recent enrichment of our domain, 'weighted algebraic topology', where paths have a weight or cost - possibly infinite (Section 12).

This paper is based on my contribution at the conference "Charles Ehresmann: 100 ans", Amiens, 7-9 October 2005, with various extensions and updates.

\section{Combinatorial Settings And DiReCted homology}

First, directed homotopy and homology can be developed for cubical sets (as in [G5, G6]) and simplicial sets.

Let us recall that a topological space $T$ has intrinsic symmetries, appearing - at the lowest level - in the reversion of its paths. Thus, the set $\Delta_{n} T=\operatorname{Top}\left(\Delta^{n}, T\right)$ of its singular simplices inherits from the standard simplex $\Delta^{n}$ an obvious action of the symmetric group $S_{n+1}$, while the set $\square_{n} T=\operatorname{Top}\left([0,1]^{n}, T\right)$ of its singular cubes has a similar action of the hyperoctahedral group (the group of symmetries of the $n$-cube). These combinatorial structures produce the singular homology of the space $T$, which can be equivalently defined as the homology of the chain complex associated to the simplicial set $\Delta T$, or the homology of the (normalised) chain complex associated to the cubical set $\square T$. We will follow the cubical approach, also to use the natural order on $\mathbf{I}^{n}$ (cf. Section 4).

Now, bypassing topological spaces, an abstract cubical set $X$ is a merely combinatorial structure, consisting of a sequence of sets $X_{n}$, with faces $\partial_{i}^{\alpha}: X_{n} \rightarrow X_{n-1}$ and degeneracies $e_{i}: X_{n-1} \rightarrow X_{n}(\alpha= \pm ; i=1, \ldots, n)$ satisfying the well-known cubical relations. This structure has been used in 
two ways, in [G5]: to break the symmetries considered above and to perform constructions, namely quotients, which would be useless in ordinary topology.

For the first aspect, note that an 'edge' in $X_{1}$ need not have any counterpart with reversed vertices, nor a 2-cube in $X_{2}$ any counterpart with horizontal and vertical faces interchanged. Thus, our structure has 'privileged directions' in any dimension (usually ignored), and the ordinary homology of $X$ can be given a preorder, generated by taking the given cubes as positive. For instance, the obvious cubical model $\uparrow \mathbf{s}^{n}$ of the n-dimensional sphere, with one non-degenerate cube in dimension $n$, has directed homology $\uparrow H_{n}\left(\uparrow \mathbf{s}^{n}\right)$ consisting of the group of integers, with the natural order. Direction should not be confused with orientation, as shown by the model $\uparrow \mathbf{t}^{2}=\uparrow \mathbf{s}^{1} \otimes \uparrow \mathbf{s}^{1}$ of the 2-dimensional torus, where $\uparrow H_{1}\left(\uparrow \mathbf{t}^{2}\right) \cong \uparrow \mathbf{Z}^{2}$ has the product order.

Secondly, it may happen that a quotient $T / \sim$ of a topological space has a trivial topology, while the corresponding quotient of its singular cubical set $\square T$ keeps a relevant topological information, detected by its homology and agreeing with the interpretation of such quotients in noncommutative geometry, as recalled in the next section.

\section{INTERACTIONS WITH NONCOMMUTATIVE GEOMETRY}

Let us start from the well-known irrational rotation $\mathrm{C}^{*}$-algebras, also known as 'noncommutative tori'.

First, take the line $\mathbf{R}$ and its (dense) additive subgroup $G_{\vartheta}=\mathbf{Z}+\vartheta \mathbf{Z}$ (with $\vartheta$ an irrational number), which acts on the line by translations. In Top, the orbit space $\mathbf{R} / G_{\vartheta}=\mathbf{S}^{1} / \vartheta \mathbf{Z}$ is trivial: an uncountable set with the coarse topology. Second, consider the Kronecker foliation $F$ of the torus $\mathbf{T}^{2}=\mathbf{R}^{2} / \mathbf{Z}^{2}$, with irrational slope $\vartheta$, and the set $\mathbf{T}_{\vartheta}^{2}=\mathbf{T}^{2} / \equiv_{F}$ of its leaves (in bijective correspondence with the previous set $\mathbf{R} / G_{\vartheta}$ ). Again, topology gives no information on $\mathbf{T}_{\vartheta}^{2}$, since all leaves are dense and the quotient space $\mathbf{T}^{2} / \equiv_{F}$ is coarse.

In noncommutative geometry, both these sets are 'interpreted' as the (noncommutative) $\mathrm{C}^{*}$-algebra $A_{\vartheta}$, generated by two unitary elements $u, v$ under the relation $v u=\exp (2 \pi i \vartheta) \cdot u v$, and called the irrational rotation algebra associated with $\vartheta$, or also a noncommutative torus $[\mathrm{Ri}, \mathrm{Co}]$. Both its complex K-theory groups are two-dimensional. These algebras have been classified, by proving that $K_{0}\left(A_{\vartheta}\right) \cong \uparrow G_{\vartheta}$ as a (totally) ordered subgroup of R. Thus, $A_{\vartheta}$ and $A_{\vartheta^{\prime}}$ are strongly Morita equivalent if and only if $\uparrow G_{\vartheta} \cong \uparrow G_{\vartheta^{\prime}}$ (as ordered groups), if and only if $\vartheta$ and $\vartheta^{\prime}$ are equivalent modulo the action of the group PGL $(2, \mathbf{Z})[\mathrm{PV}, \mathrm{Ri}]$.

Now, for a group $G$ acting properly on an acyclic space $T$, a classical result says that the homology of the orbit space $T / G$ is isomorphic to the homology of the group $G$; these results can be extended to free actions if we replace the space $T$ with its singular cubical set $\square T$ and take the quotient 
cubical set $(\square T) / G$ ([G5], Thm. 3.3). Thus, the trivial orbit space $\mathbf{R} / G_{\vartheta}$ can be replaced with a non-trivial cubical set, $(\square \mathbf{R}) / G_{\vartheta}$, whose homology is the same as the homology of the group $G_{\vartheta} \cong \mathbf{Z}^{2}$, and coincides with the homology of the torus $\mathbf{T}^{2}$. Algebraically, all this is in accord with the noncommutative $\mathrm{C}^{*}$-algebra $A_{\vartheta}$, but our result is independent of $\vartheta$ (as soon as $\vartheta$ is irrational) and does not allow us to recover it, at any extent.

This becomes possible with directed homology. The quotient $(\square \mathbf{R}) / G_{\vartheta}$ can be modified, replacing $\square \mathbf{R}$ with the cubical set $\square \uparrow \mathbf{R}$ of the directed line, formed of all order-preserving maps $\mathbf{I}^{n} \rightarrow \mathbf{R}$. Algebraically, the homology groups are unchanged, but now $\uparrow H_{1}\left((\square \uparrow \mathbf{R}) / G_{\vartheta}\right) \cong \uparrow G_{\vartheta}$ as an ordered subgroup of $\mathbf{R}$ ([G5], Thm. 4.8): thus the rotation cubical sets $C_{\vartheta}=(\square \uparrow \mathbf{R}) / G_{\vartheta}$ have the same classification up to isomorphism ([G5], Thm. 4.9) as the rotation $\mathrm{C}^{*}$-algebras $A_{\vartheta}$ up to strong Morita equivalence, and $\vartheta$ is again determined up to the action of $\operatorname{PGL}(2, \mathbf{Z})$. This example shows that the ordering of directed homology can carry a relevant information, even much finer than the algebraic one. (The inequilogical spaces $C_{\vartheta}^{\prime}=\left(\uparrow \mathbf{R}, \equiv_{G_{\vartheta}}\right)$ give the same results, cf. Section 9.)

Further, comparison with the stricter classification of the algebras $A_{\vartheta}$ up to isomorphism shows that cubical sets provide a sort of 'noncommutative topology', without the metric character of noncommutative geometry. To add this character to the present frame, one should enrich cubical sets with a sort of norm - and turn Directed into Weighted Algebraic Topology (see [G6] and Section 12).

\section{A Basic topological SETting}

After the combinatorial setting considered above, the simplest topological situation where one can study directed paths and directed homotopies is likely the category p Top of preordered topological spaces and preorderpreserving continuous mappings. (Here, a preorder relation is reflexive and transitive; it is an order if it is also anti-symmetric.)

In this setting, a (directed) path of the preordered space $X$ is a morphism $a: \uparrow[0,1] \rightarrow X$, defined on the standard directed interval $\uparrow \mathbf{I}=\uparrow[0,1]$, with euclidean topology and natural order. A (directed) homotopy $\varphi: f \rightarrow$ $g: X \rightarrow Y$, from $f$ to $g$, is a map $\varphi: X \times \uparrow \mathbf{I} \rightarrow Y$ coinciding with $f$ on the lower basis of the cylinder $I(X)=X \times \uparrow \mathbf{I}$, with $g$ on the upper one. Of course, this (directed) cylinder is a product in pTop : it is equipped with the product topology and with the product preorder, where $(x, t) \prec\left(x^{\prime}, t^{\prime}\right)$ if $x \prec x^{\prime}$ in $X$ and $t \leq t^{\prime}$ in $\uparrow \mathbf{I}$.

The category pTop has all limits and colimits, constructed as in Top and equipped with the initial or final preorder for the structural maps. The forgetful functor $U:$ pTop $\rightarrow$ Top, with values in the category of topological spaces, has both a left and a right adjoint, $D \dashv U \dashv C$, where $D X$ (resp. $C X)$ is the space $X$ with the discrete order (resp. the coarse preorder). The standard embedding of Top in pTop will be the coarse one, so that all 
(ordinary) paths in $X$ are directed in $C X$. Note that the category of ordered spaces does not allow for such an embedding and would not allow one to view classical Algebraic Topology within the Directed one; moreover, it has different colimits, not consistent with the topological ones.

The fundamental category $C=\uparrow \Pi_{1}(X)$ has, for arrows, the classes of directed paths up to the equivalence relation generated by directed homotopy with fixed endpoints; composition is given by the concatenation of consecutive paths. The fundamental category of a preordered space can be computed by a van Kampen-type theorem, as proved in [G4], Thm. 3.6, in a much more general setting ('d-spaces', cf. Section 9). The obvious functor $\uparrow \Pi_{1}(X) \rightarrow \Pi_{1}(U X)$ with values in the fundamental groupoid of the underlying space need neither be full (obviously), nor faithful. As a typical component of directed algebraic topology, a preordered space $X$ has an opposite objet $R X=X^{\mathrm{op}}$, with the reversed preorder, and $\uparrow \Pi_{1}\left(X^{\mathrm{op}}\right)$ is the opposite category $\left(\uparrow \Pi_{1}(X)\right)^{\text {op }}$.

A map $f: X \rightarrow Y$ induces a functor $f_{*}: \uparrow \Pi_{1}(X) \rightarrow \uparrow \Pi_{1}(Y)$, and a homotopy $\varphi: f \rightarrow g$ induces a natural transformation $\varphi_{*}: f_{*} \rightarrow g_{*}$ which generally is not invertible. Also because of this, there are crucial differences with the fundamental groupoid $\Pi_{1}(S)$ of a space, for which a model up to homotopy invariance is given by the skeleton: a family of fundamental groups $\pi_{1}\left(S, x_{i}\right)$, obtained by choosing one point in each path-connected component of $S$. For instance, if $X$ is ordered, the fundamental category has no isomorphisms nor endomorphisms, except the identities. Thus: (a) the category is skeletal, and ordinary equivalence of categories cannot yield any simpler model; (b) all the monoids $\uparrow \pi_{1}\left(X, x_{0}\right)=\uparrow \Pi_{1}(X)\left(x_{0}, x_{0}\right)$ are trivial and give no information on the fundamental category.

Similarly, the singular cubical set $\square X$ consists of all preorder-preserving maps $\uparrow \mathbf{I}^{n} \rightarrow X$, and provides the preordered homology groups $\uparrow H_{n}(X)$ (studied in [G7] in a more general setting). All this works because the faces and degeneracies $\uparrow \mathbf{I}^{n-1} \rightleftarrows \uparrow \mathbf{I}^{n}$ of the ordered cubes preserve the natural orders; it is not clear how this construction might be transferred to tetrahedra (consistently with barycentric subdivision).

\section{Analysing the fundamental Category}

An elementary example will give some idea of the analysis developed in [G8] for the fundamental category of a preordered space. (The paper [FRGH], devoted to the analysis of concurrent processes, has similar results, based on different categorical tools: categories of fractions; see also [GH], in this volume.)

Let us start from the standard ordered square $\uparrow[0,1]^{2}$, with the euclidean topology and the product order, and consider the (compact) ordered subspace $A$ obtained by taking out the open square $] 1 / 3,2 / 3[2$, a sort of 'square 
annulus'

(1)

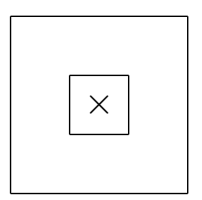

$A$

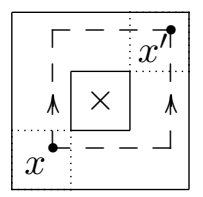

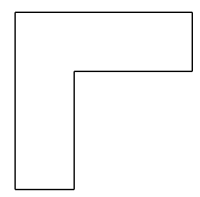

$L$

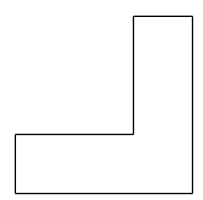

$L^{\prime}$

Its directed paths, the continuous order-preserving maps $\uparrow[0,1] \rightarrow A$, move 'rightward and upward'. The fundamental category $C=\uparrow \Pi_{1}(A)$ has some arrow $x \rightarrow x^{\prime}$ provided that $x \leq x^{\prime}$ and both points are in $L$ or $L^{\prime}$ (the closed subspaces represented above): there are two arrows when $x \leq p=(1 / 3,1 / 3)$ and $x^{\prime} \geq q=(2 / 3,2 / 3)$, and one otherwise. This evident fact can be easily proved with the 'van Kampen' theorem recalled above, using the subspaces $L, L^{\prime}$ (whose fundamental category is the induced order).

Thus, the whole category $C$ is easy to visualise and 'essentially represented' by the full subcategory $E$ on four vertices $0, p, q, 1$ (the central cell does not commute)
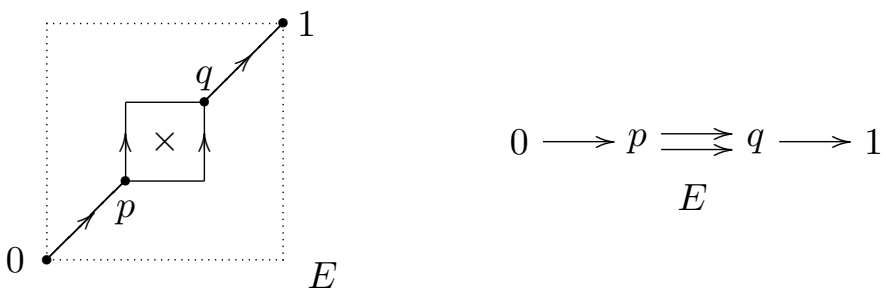

$E$

But $E$ is far from being equivalent to $C$, as a category, since $C$ is already a skeleton, in the ordinary sense. In [G8] we have introduced two (dual) directed notions, which take care, respectively, of variation 'in the future' or 'from the past': future equivalence (a symmetric version of an adjunction, with two units, see the next section) and its dual, a past equivalence (with two counits); and studied how to extract minimal models for both relations and how to combine them.

In the present case, $C$ has a minimal 'future model' $F$ (the least full reflective subcategory) and a minimal 'past model' $P$ (coreflective). The full subcategory $E$ is the join of $F$ and $P$; it is at the same time future 
equivalent and past equivalent to $C$, and a 'minimal injective model' of $C$.
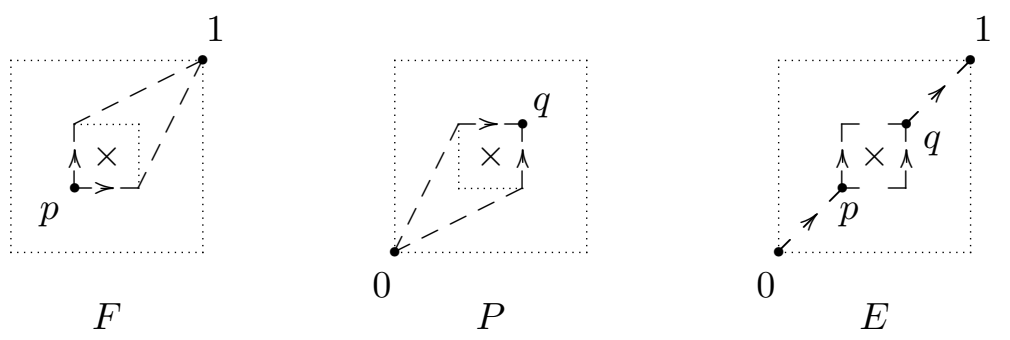

Now, the process represented by the ordered space $X$ can be analysed as follows, in the finite model $E$ :

- the action begins at 0 , from where we move to the point $p$,

- $p$ is an (effective) future branching point, where we have to choose between two paths,

- which join at $q$, an (effective) past branching point,

- from where we can only move to 1 , where the process ends.

More complex examples can be found in [G8, FRGH, GH].

\section{Directed EQuivalences of CATEGORIES}

Directed homotopy equivalence is a complex notion, which can be developed in various ways - both for spaces and their algebraic models. Various such notions for categories have been developed in [G8]; here we only give a partial review. (For an investigation of this notion for directed spaces see [Ra], in this volume.)

A future equivalence $(f, g ; \varphi, \psi)$ between the categories $C, D([\mathrm{G} 8], 2.1)$ is a 'symmetric version' of an adjunction, with two units. It consists of a pair of functors and a pair of natural transformations (i.e., directed homotopies in Cat), the units, satisfying two coherence conditions:

$$
\begin{gathered}
f: C \rightleftarrows D: g \quad \varphi: 1_{C} \rightarrow g f, \quad \psi: 1_{D} \rightarrow f g, \\
f \varphi=\psi f: f \rightarrow f g f, \quad \varphi g=g \psi: g \rightarrow g f g \quad \text { (coherence). }
\end{gathered}
$$

Note that the directed homotopies $\varphi, \psi$ proceed from the identities to the composites $g f, f g$ ('in the future'). Future equivalences compose (much in the same way as adjunctions), and yield an equivalence relation of categories.

An adjunction $f \dashv g$ with invertible counit $\varepsilon: f g \cong 1$ amounts to a future equivalence with invertible $\psi=\varepsilon^{-1}$. In this case, a 'split' future equivalence, $D$ can be identified with a full reflective subcategory of $C$ (also called a future retract). But, in a general future equivalence, $f$ need not determine $g$. Theorem 2.5, in [G8], shows that two categories are future equivalent if and only if they can be embedded into a common one, as full reflective subcategories; the latter is explicitly constructed. 
Dually, past equivalences have counits, in the opposite direction. These two basic notions can be combined in various ways, to give various selfdual equivalence relations. First, their conjunction is called past and future equivalence, while coarse equivalence is the equivalence relation generated by them (or, equivalently, by the existence of an adjunction between two categories, as one can easily deduce from two factorisation theorems of [G8], 2.5 and 4.4 ).

More complex and interesting combinations, where a functor $C \rightarrow D$ is at the same time a future and a past equivalence, with (generally) different 'quasi-inverses', are injective equivalences and projective equivalences (see [G8], 4.1).

Correspondingly, we have various notions of contractibility in Cat. First, it is easy to prove that a category is future contractible (i.e. future equivalent to the singleton category $\mathbf{1}$ ) if and only if it has a terminal object ([G8], 2.6); dually, it is past contractible if and only if it has an initial object.

Furthermore, a category is injectively contractible (i.e., injectively equivalent to $\mathbf{1}$ ) if and only if it has a zero object ([G8], 5.4). On the other hand, a category with non-isomorphic initial and terminal object is injectively equivalent to the ordinal $\mathbf{2}=\{0 \rightarrow 1\}$; the latter is the standard directed interval of Cat (and is not injectively contractible). Finally, a category is projectively equivalent to $\mathbf{1}$ if and only if it has initial and terminal objects (isomorphic or not), which amounts to being past contractible and future contractible ([G8], 5.4). But, in general, injective equivalence is stronger than past and future equivalence.

\section{TWO-DIMENSIONAL ANALYSIS}

In [G9], we have extended this analysis of the fundamental category of a preordered space $X$ (or, more generally, of a d-space) introducing a strict fundamental 2-category $\uparrow \Pi_{2}(X)$. But this construction is complicated, perhaps non natural, and it is not clear whether it can be extended to higher dimension.

More naturally, one can define a fundamental biased d-lax 2-category $\uparrow \mathrm{b} \Pi_{2}(X)$, as studied in [G10]. It is interesting to note that the geometric guideline gives precise directions for the comparison cells, different from the ones previously considered, by Burroni $[\mathrm{Br}]$ and Leinster [Le] for lax 2-categories (biased and unbiased, respectively). The term 'd-lax' refers to this choice, while the term 'biased' or 'unbiased' refers to structures based on binary or multiple operations, respectively.

An object of $\uparrow \mathrm{b} \Pi_{2}(X)$ is a point of $X$, an arrow $a: x \rightarrow x^{\prime}$ is a path, and a cell $[\alpha]: a \rightarrow a^{\prime}: x \rightarrow x^{\prime}$ is a homotopy class of homotopies of paths. More precisely, $\alpha$ is a 2-homotopy $a \rightarrow a^{\prime}$ (with fixed endpoints), and its class $[\alpha]$ is up to the equivalence relation generated by 3-homotopies $\alpha^{6} \rightarrow \alpha^{\prime \prime}$ (with fixed boundary). 
Now, writing $a \otimes b$ the concatenation of two consecutive paths, our structure has comparison cells, for units and associativity, directed as follows:

$$
\lambda: 1_{x} \otimes a \rightarrow a, \quad \rho: a \rightarrow a \otimes 1_{x^{\prime}}, \quad \kappa: a \otimes(b \otimes c) \rightarrow(a \otimes b) \otimes c
$$

always going from a first concatenation to a second concatenation of the same paths which, at each instant, has made a longer way than the initial one.

Then, the coherence theorem for such a structure says that all diagrams (naturally) constructed with comparison cells commute. This remains true in an extended structure, where we add higher associativity comparisons $\kappa^{\prime}, \kappa^{\prime \prime}$ depending on four consecutive arrows, which break Mac Lane's pentagon into 3 commutative triangles:

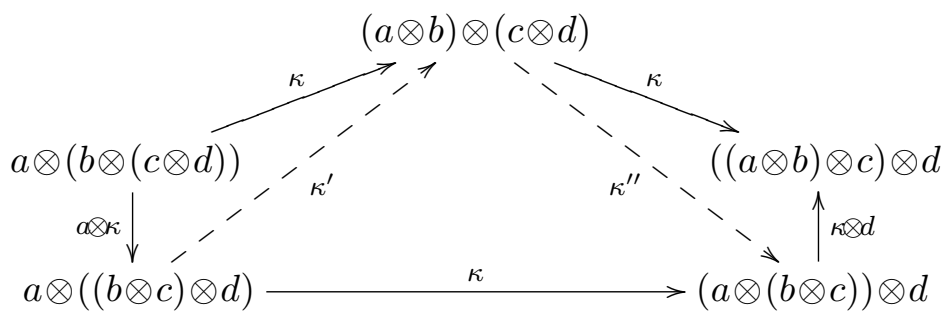

There is also a larger unbiased version, $\uparrow u \Pi_{2}(X)$, with $n$-ary concatenations $a_{1} \otimes \ldots \otimes a_{n}$ of consecutive paths ([G10], Section 3). Finally, provided that $\mathrm{X}$ has a $T_{1}$-topology, a further extension, studied in [G11], gives a fundamental absolute (unbiased) d-lax 2-category $\uparrow \mathrm{L}_{2}(X)$, with the same objects, arrows and cells as $\uparrow u \Pi_{2}(X)$, but having extended absolute comparisons $\varphi(a, b): a \rightarrow b$, which only depend on the actual values of the paths $a, b$ rather than on their 'syntactic construction'.

\section{LINKS WITH CATEGORICAL MODELS FOR BIOLOGICAL SYSTEMS}

The analysis of a category through minimal past and future models, as sketched in Section 5 and developed in [G8], has appeared to be closely related with notions recently introduced by A.C. Ehresmann [Eh], aiming to model biological and neural systems. Likely, because of the common design of studying non-reversible actions.

It would be difficult to fully explain this here. Let us only remark two pairs of neighbouring notions, using the terminology of both papers. First, a past retract $P$ of a category $X$ (i.e. a full coreflective subcategory), used in [G8] as a 'past model' of $X$, is plainly a particular case of a corefract (i.e. a full weakly coreflective subcategory) as defined in [Eh] 1.2. Second, one can prove that the past spectrum $P$ of a category $X$ having no $O^{-}$-branching points, in the sense of [G8], is necessarily a root of $X$, as defined in [Eh], Section 2. In [G8], many examples of Section 9 fall in this situation: their past spectrum is a root and their future spectrum a co-root. 


\section{Richer topologicAl SETTINGS}

In a preordered space, every loop lives in a zone where the preorder is chaotic, and is reversible; therefore, this setting has no 'directed circle' or 'directed torus'.

We briefly recall more complex directed structures, which allow for nonreversible loops. All of them contain the directed interval $\uparrow \mathbf{I}$, so that all the previous constructions can be easily extended.

(a) In a setting studied in [G4], a $d$-space $X$ is a topological space equipped with a set $d X$ of (continuous) maps $a: \mathbf{I} \rightarrow X$; these maps, called $d i$ rected paths or $d$-paths, must contain all constant paths and be closed under concatenation and (weakly) increasing partial reparametrisation. A d-map $f: X \rightarrow Y$ (or map of d-spaces) is a continuous mapping between d-spaces which preserves the directed paths: if $a \in d X$, then $f a \in d Y$.

The category of d-spaces is written as dTop. It has all limits and colimits, constructed as in Top and equipped with the initial or final d-structure for the structural maps. Again, the forgetful functor $U: \mathrm{d}$ Top $\rightarrow$ Top has a left and a right adjoint; a topological space is viewed as a d-space by its natural structure, where all (continuous) paths are directed (via the right adjoint to $U$ ). Also pTop has an obvious functor with values in dTop.

Reversing d-paths, by the involution $r(t)=1-t$, yields the reflected, or opposite, d-space $R X=X^{\mathrm{op}}$. The standard directed circle $\uparrow \mathbf{S}^{1}=\uparrow \mathbf{I} / \partial \mathbf{I}$ has the obvious d-structure, where paths have to follow a precise orientation.

(b) Another setting for Directed Algebraic Topology comes from a directed version of Dana Scott's equilogical spaces [Sc, BBS], which was introduced in $[\mathrm{G} 7]$.

An inequilogical space $X=\left(X^{\sharp}, \sim_{X}\right)$ is a preordered topological space $X^{\sharp}$ endowed with an equivalence relation $\sim_{X}$. The quotient $|X|=X^{\sharp} / \sim_{X}$ is viewed as a preordered topological space (with the induced preorder and topology), or a topological space, or a set, as convenient. A map $f: X \rightarrow$ $Y$ 'is' a mapping $f:|X| \rightarrow|Y|$ which admits some continuous preorderpreserving lifting $f^{\prime}: X^{\sharp} \rightarrow Y^{\sharp}$.

This category is denoted as pEql. The category p Top fully embeds in the latter, identifying a preordered space $X$ with the pair $\left(X,=_{X}\right)$. The new category has all limits and colimits, and is cartesian closed (like the one of equilogical spaces). Directed homotopy is defined by the standard directed interval $\uparrow \mathbf{I}$. Various models for the directed circle are considered in [G7]; the simplest is perhaps $(\uparrow \mathbf{R}, \equiv \mathbf{z})$, i.e. the quotient in $\mathrm{pEq}$ of the directed real line modulo the action of the group of integers.

(c) Recently, S. Krishnan $[\mathrm{Kr}]$ has proposed a 'convenient category of locally preordered spaces' which, in contrast with the previous versions of this notion, has all colimits and therefore allows for the usual constructions of homotopy theory, like mapping cones and suspension. 
10. The Beginning of a formal setting for Directed Algebraic TOPOLOGY

The phenomena we are studying make sense in a category $\mathbf{A}$ equipped with 2-cells (homotopies) which, generally, cannot be reversed - but reflected.

More precisely, as a variation of Kan's notion of a category equipped with an abstract cylinder endofunctor [Ka], $\mathbf{A}$ is a dI1-category. By this we mean that it comes equipped with:

(a) a reflector $R: \mathbf{A} \rightarrow \mathbf{A}$, i.e. an involutive (covariant) automorphism (also written $\left.R(X)=X^{\mathrm{op}}, R(f)=f^{\mathrm{op}}\right)$,

(b) a cylinder endofunctor $I: \mathbf{A} \rightarrow \mathbf{A}$, with four natural transformations: two faces $\left(\partial^{\alpha}\right)$, a degeneracy $(e)$ and a reflection $(r)$

$$
\partial^{\alpha}: \mathrm{id} \rightleftarrows I: e, \quad r: I R \rightarrow R I \quad(\alpha= \pm),
$$

satisfying the equations

$$
\begin{array}{ll}
e \partial^{\alpha}=1: \mathrm{id} \rightarrow \mathrm{id}, & R r R . r=1: I R \rightarrow I R, \\
R e . r=e R: I R \rightarrow R, & r . \partial^{-} R=R \partial^{+}: R \rightarrow R I .
\end{array}
$$

Since $R R=1$, the transformation $r$ is invertible with $r^{-1}=R r R: R I \rightarrow$ $I R$ and $r . \partial^{+} R=R \partial^{-}$.

A homotopy $\varphi: f^{-} \rightarrow f^{+}: X \rightarrow Y$ is defined as a map $\varphi: I X \rightarrow Y$ with $\varphi \cdot \partial^{\alpha} X=f^{\alpha}$ (also written $\hat{\varphi}$ to distinguish it from the homotopy). Each map $f: X \rightarrow Y$ has a trivial endohomotopy, $0_{f}: f \rightarrow f$, represented by f.eX $=e Y . I f: I X \rightarrow Y$.

Every homotopy $\varphi: f \rightarrow g: X \rightarrow Y$ has a reflected homotopy

$$
\varphi^{\mathrm{op}}: g^{\mathrm{op}} \rightarrow f^{\mathrm{op}}: X^{\mathrm{op}} \rightarrow Y^{\mathrm{op}}, \quad\left(\varphi^{\mathrm{op}}\right)^{\wedge}=R(\hat{\varphi}) \cdot r: I R X \rightarrow R Y,
$$

and $\left(\varphi^{\mathrm{op}}\right)^{\mathrm{op}}=\varphi,\left(0_{f}\right)^{\mathrm{op}}=0_{\left(f^{\mathrm{op}}\right)}$.

An object $X$ is said to be reversible if it coincides with $X^{\mathrm{op}}$, and reflexive or self-dual if it is isomorphic to the latter. (The setting itself is reversible when $R=i d \mathbf{A}$; then, a homotopy has a reversed homotopy $\varphi^{\mathrm{op}}: g \rightarrow f$. Such settings have no 'privileged directions'.)

Dually, a dP1-category has a reflection (as above) and a cocylinder, or path endofunctor $P: \mathbf{A} \rightarrow \mathbf{A}$, with natural transformations in the opposite direction

$$
e: \text { id } \leftrightarrows P: \partial^{\alpha}, \quad r: R P \rightarrow P R .
$$

satisfying the dual axioms.

It is easy to see that a dI1-structure where the cylinder functor has a right adjoint, $I \dashv P$, automatically produces the natural transformations of the cocylinder, and a dP1-structure; we say then that $\mathbf{A}$ is a dIP1-category. Both endofunctors produce the same homotopies, equivalently represented by maps $I X \rightarrow Y$ or $X \rightarrow P Y$.

A dI1- or dP1-structure is often generated by a 'standard directed interval' (equipped with faces and degeneracy), by cartesian (or tensor) product and 
internal hom, respectively. In these frames, one can define directed homology and 'first order' homotopy theory.

\section{Higher StRuCture}

Of course, much structure has to be added to develop higher directed homotopy theory. In part, this has already be done in the 90's [G1, G2, G3], but these papers were focused on the reversible case, even if taking into account more general situations and some non-reversible examples (especially [G2]). They need to be rethought, at the light of the recent ideas and developments of Directed Algebraic Topology.

A main ingredient will certainly be the notion of a cubical monad with reflection and interchange $\left(\mathbf{A}, R, I, \partial^{\alpha}, e, r, g^{\alpha}, s\right)$, already present in [G2]. In many cases (like preordered spaces, d-spaces and inequilogical spaces) the new structure derives from the lattice structure of the euclidean interval, with the binary operations $g^{-}, g^{+}$

$$
\begin{aligned}
& g^{\alpha}:[0,1]^{2} \rightarrow[0,1], \\
& g^{-}\left(t, t^{\prime}\right)=\max \left(t, t^{\prime}\right), \quad g^{+}\left(t, t^{\prime}\right)=\min \left(t, t^{\prime}\right),
\end{aligned}
$$

which commute with respect to the interchange of coordinates, $s:[0,1]^{2} \rightarrow$ $[0,1]^{2}$.

Formally, we are adding to the previous structure of dI1-category (Section 10), three natural transformations: the lower and upper connections $g^{-}, g^{+}$ and the interchange s. Globally, we have now seven natural transformations $\partial^{\alpha}, e, r, g^{\alpha}, s$ (with $\alpha= \pm$ )

$$
\text { id } \underset{e}{\stackrel{\partial^{\alpha}}{\rightleftarrows}} I \stackrel{g^{\alpha}}{\rightleftarrows} I^{2} \quad I^{2} \stackrel{s}{\longrightarrow} I^{2} \quad I R \stackrel{r}{\longrightarrow} R I
$$

under the following axioms (which include the ones of dI1-categories)

$$
\begin{aligned}
& e \partial^{\alpha}=1, \quad e g^{\alpha}=e . I e(=e . e I) \quad \text { (degeneracy), } \\
& g^{\alpha} \cdot I g^{\alpha}=g^{\alpha} \cdot g^{\alpha} I, \quad g^{\alpha} \cdot I \partial^{\alpha}=1=g^{\alpha} \cdot \partial^{\alpha} I \quad \text { (associativity, unit), } \\
& g^{\beta} . I \partial^{\alpha}=\partial^{\alpha} e \quad g^{\beta} . \partial^{\alpha} I=\partial^{\alpha} e \quad \text { (absorbency; } \alpha \neq \beta \text { ), } \\
& \text { RrR.r }=1, \quad \text { Re.r }=e R, \\
& r . \partial^{-} R=R \partial^{+}, \quad r . g^{+} R=R g^{-} . r_{2}, \\
& \text { s.s }=1, \quad I \text { e.s }=e I \text {, } \\
& \text { s.I } \partial^{\alpha}=\partial^{\alpha} I, \quad g^{\alpha} . s=g^{\alpha} \text {, } \\
& R R=1, \quad R s . r_{2}=r_{2} . s R \quad \text { (symmetries). }
\end{aligned}
$$

Here, $r_{2}=r I . I r:\left(I^{2} R \rightarrow I R I \rightarrow R I^{2}\right)$ is the reversion of the double cylinder.

(A cubical monad corresponds to a simple algebraic structure, which extends a lattice with minimum and maximum: a cubical monoid is a set with two monoid structures, such that the identity of each operation is an absorbent element for the other operation. Thus, a monoid is to a cubical monoid what a monad is to a cubical monad, or what an augmented simplicial set is to cubical set [G1, G2].) 
Now, we have to add structure and axioms for concatenation; this requires some care, in order to include the previous settings (and others), and cannot be sketched here.

\section{From diRECTEd TO Weighted Algebraic TOPOLOGY}

We end with some hints to a quite recent enrichment of the main subject of this review, weighted algebraic topology, which replaces the two-valued logic of directed algebraic topology (where a path is licit or not) with a measure of costs, taking values in the interval $[0, \infty]$ of positive real extended numbers and marking illicit paths with an infinite cost.

The general aim can now be expressed as: measuring the cost of phenomena, where cost can mean energy, price, duration, etc., and is not assumed to be invariant under direction. Weighted algebraic topology will study 'weighted spaces', using 'weighted' algebraic structures, like the fundamental weighted (or seminormed) category [G12], and the weighted (or seminormed) homology groups already developed in [G6] for normed cubical sets.

Lawvere's generalised metric spaces $[\mathrm{Lw}]$, which are endowed with a (generally) non-symmetric distance taking values in $[0, \infty]$, are a prime setting where weighted algebraic topology can be developed. Now, homotopies are based on the standard generalised metric interval $\delta \mathbf{I}$, with distance $\delta(x, y)=y-x$, if $x \leq y$, and $\delta(x, y)=\infty$ otherwise; the cylinder functor is $I(X)=X \otimes \delta \mathbf{I}$, where the tensor product is the cartesian product of the underlying sets with the $l_{1}$-type metric. This allows us to define the fundamental weighted category $\mathrm{w}_{1}(X)$ of a generalised metric space, and begin its study [G12].

Note that the previous formal setting (Sections 10,11) applies also in this situation, with $X^{\mathrm{op}}$ equipped with the opposite distance $\delta^{\mathrm{op}}(x, y)=$ $\delta(y, x)$ and the natural transformations of the cylinder defined by the 'same' structure on $\delta \mathbf{I}$.

We also introduce, in the same article, the more flexible setting of $w$ spaces, or spaces with weighted paths, which has finer quotients and is able to express irrational rotation structures, as previously done with cubical sets (see Section 3) and - even more similarly - with the normed cubical sets of [G6].

\section{REFERENCES}

[BBS] A. Bauer, L. Birkedal and D.S. Scott, Equilogical spaces, Theoretical Computer Science 315 (2004), 35-59.

[Br] A. Burroni, T-catgories, Cah. Topol. Gom. Diffr. 12 (1971), 215-321.

[Co] A. Connes, Noncommutative geometry, Academic Press, San Diego CA 1994.

[Eh] A.C. Ehresmann, Localization of universal problems. Local colimits, Appl. Categ. Structures 10 (2002), 157-172.

[FRGH] L. Fajstrup, M. Raussen, E. Goubault and E. Haucourt, Components of the fundamental category, Appl. Categ. Structures 12 (2004), 81-108.

[Ga] P. Gaucher, A model category for the homotopy theory of concurrency, Homology Homotopy Appl. 5 (2003), 549-599. 
[Go] E. Goubault, Geometry and concurrency: a user's guide, in: Geometry and concurrency, Math. Structures Comput. Sci. 10 (2000), no. 4, pp. 411-425.

[GH] E. Goubault and E. Haucourt, Components of the fundamental category II, Appl. Categ. Structures, this issue.

[G1] M. Grandis, Cubical monads and their symmetries, in: Proceedings of the Eleventh International Conference on Topology, Trieste 1993, Rend. Ist. Mat. Univ. Trieste, 25 (1993), 223-262.

[G2] M. Grandis, Cubical homotopical algebra and cochain algebras, Ann. Mat. Pura Appl. 170 (1996) 147-186.

[G3] M. Grandis, Categorically algebraic foundations for homotopical algebra, Appl. Categ. Structures 5 (1997), 363-413.

[G4] M. Grandis, Directed homotopy theory, I. The fundamental category, Cah. Topol. Gom. Diffr. Catg. 44 (2003), 281-316.

[G5] M. Grandis, Directed combinatorial homology and noncommutative tori (The breaking of symmetries in algebraic topology), Math. Proc. Cambridge Philos. Soc. 138 (2005), 233-262.

[G6] M. Grandis, Normed combinatorial homology and noncommutative tori, Theory Appl. Categ. 13 (2004), No. 7, 114-128.

[G7] M. Grandis, Inequilogical spaces, directed homology and noncommutative geometry, Homology Homotopy Appl. 6 (2004), 413-437.

[G8] M. Grandis, The shape of a category up to directed homotopy, Theory Appl. Categ. 15 (2005) (CT2004), No. 4, 95-146.

[G9] M. Grandis, Modelling fundamental 2-categories for directed homotopy, Homology Homotopy Appl. 8 (2006), 31-70.

[G10] M. Grandis, Lax 2-categories and directed homotopy, Cah. Topol. Gom. Diff. Catg., to appear. Preprint: Dip. Mat. Univ. Genova, Preprint 530 (2005). http://www.dima.unige.it/ grandis/LCat.pdf

[G11] M. Grandis, Absolute lax 2-categories, Appl. Categ. Struct. 14 (2006), 191-214.

[G12] M. Grandis, The fundamental weighted category of a weighted space (From directed to weighted algebraic topology), Dip. Mat. Univ. Genova, Preprint 540 (2006). http://www.dima. unige.it/ grandis/wHtp.pdf

[Ka] D.M. Kan, Abstract homotopy I, Proc. Nat. Acad. Sci. U.S.A. 41 (1955), 1092-1096.

[Kr] S. Krishnan, A convenient category of locally ordered spaces, presented at GETCO 2005. Unpublished Manuscript. http://www.math.uchicago.edu/ sanjeevi/

[La] Y. Lafont, Algebra and geometry of rewriting, Appl. Categ. Structures, this issue.

[Le] T. Leinster, Higher operads, higher categories, Cambridge University Press, Cambridge 2004.

[Lw] F.W. Lawvere, Metric spaces, generalized logic and closed categories, Rend. Sem. Mat. Fis. Univ. Milano 43 (1974), 135-166. Republished in: Reprints Th. Appl. Categ. 1 (2002), 1-37. http://www.tac.mta.ca/tac/reprints/articles/1/tr1.pdf

$[\mathrm{PV}]$ M. Pimsner and D. Voiculescu, Imbedding the irrational rotation $\mathrm{C}^{*}$-algebra into an AF-algebra, J. Operator Th. 4 (1980), 93-118.

[Ra] M. Raussen, Invariants of directed spaces, Appl. Categ. Structures, this issue.

[Ri] M.A. Rieffel, $C^{*}$-algebras associated with irrational rotations, Pacific J. Math. 93 (1981), 415-429.

[Sc] D. Scott, A new category? Domains, spaces and equivalence relations, Unpublished manuscript (1996). http://www.cs.cmu.edu/Groups/LTC/

Dipartimento di Matematica, Università di Genova, Via Dodecaneso 35 , 16146-GENOVA, ITALY

E-mail address: grandis@dima.unige.it 